\title{
Composição e estrutura de uma restinga arbustiva aberta no norte do Espírito Santo e relações florísticas com formações similares no Sudeste do Brasil
}

\author{
Composition and structure of an open scrub restinga of northern Espírito Santo \\ and floristic relationships with similar formations in southeastern Brazil
}

\author{
Mariana Maciel Monteiro ${ }^{1,3}$, Augusto Giaretta ${ }^{2}$, Oberdan José Pereira ${ }^{1}$ \\ $\&$ Luis Fernando Tavares de Menezes ${ }^{1}$
}

\begin{abstract}
Resumo
Foram analisadas a estrutura e a composição florística de uma formação de restinga arbustiva aberta no Parque Estadual de Itaúnas, ES, e verificada a similaridade entre a flora das formações de restinga arbustiva da Região Sudeste do Brasil. Para o levantamento, foi utilizado o método de intercepto de linha, contemplando indivíduos com altura igual ou superior a $50 \mathrm{~cm}$. Os parâmetros fitossociológicos de frequência e valor de importância (VI) foram calculados. A similaridade da flora das restingas arbustivas da Região Sudeste foi verificada utilizando o índice de Sørensen. Foram encontradas 42 espécies em 28 famílias, sendo Fabaceae e Bromeliaceae as mais ricas (quatro espécies cada). As 10 espécies mais importantes representaram 65,6\% do VI total, evidenciando forte estrutura oligárquica, principalmente no estrato herbáceo. Assim, puderam ser apontadas espécies que caracterizam esta formação, a qual é associada com fatores ambientais que condicionam a composição e abundância florística. A análise de similaridade evidenciou que as restingas do sudeste formam dois blocos florísticos distintos, um ao norte (litoral do Espírito Santo) e outro mais ao sul (litoral do Rio de Janeiro). Foram comuns a todas as restingas analisadas Manilkara subsericea, Pilosocereus arrabidae e Byrsonima sericea, podendo ser consideradas de ampla distribuição na costa sudeste.

Palavras-chave: restinga aberta de moitas, fitossociologia, intercepto de linha, planície arenosa, Pleistoceno.
\end{abstract}

\begin{abstract}
This study analyzed the structure and floristic composition of an open scrub restinga at Parque Estadual de Itaúnas, Espírito Santo and verified the similarity between floras of the restinga formations in southeastern Brazil. The line intercept method was applied to sample individuals equal or greater than $50 \mathrm{~cm}$ tall. The frequency and an importance value were estimated. The similarity of the flora of the restinga formations in southeastern Brazil was performed using the Sørensen index. We surveyed 42 species in 28 families, being Fabaceae and Bromeliaceae the richest families (four species each). The 10 most important species represented $65.6 \%$ of the total importance value, showing a strong oligarchic structure, especially in the herbaceous layer. Thus, we identified some species that characterize this formation, which is associated with environmental factors that constrain floristic composition and abundance. The similarity analysis evidenced two distinct floristic blocks of southeastern restingas, one in the north (the coast of Espírito Santo) and another further to the south (coast of Rio de Janeiro). Some species were common to all analyzed resting formations - Manilkara subsericea, Pilosocereus arrabidae and Byrsonima sericea and may be considered of wide distribution on the southeast coast.
\end{abstract}

Key words: open scrub restinga, phytossociology, line intercept method, sandy coastal plains, Pleistocene.

\footnotetext{
${ }_{1}^{1}$ Laboratório de Ecologia de Restinga e Mata Atlântica - PPGBT, Centro Universitário Norte do Espírito Santo/UFES. BR 101 Norte, km 60, Bairro Litorâneo, 29932-540, São Mateus, ES, Brasil.

${ }^{2}$ Instituto de Pesquisas Jardim Botânico do Rio de Janeiro, Escola Nacional de Botânica Tropical, Programa de Pós-graduação em Botânica, R. Pacheco Leão 2040, 22460-036, Rio de Janeiro, RJ, Brasil.

${ }^{3}$ Autor para correspondência: marimonteiro199@gmail.com
} 


\section{Introdução}

A restinga é composta por formações vegetais florística e estruturalmente diferenciadas distribuídas ao longo do litoral brasileiro, sobre planícies arenosas construídas através de processos eólicos ou fluvio-marinhos durante o Quaternário (Suguio \& Tessler 1984; Martin et al. 1997). A variedade de formações vegetais encontrada na restinga está estreitamente ligada à variação geomorfológica e de outras condições ambientais associadas, incluindo variação na topografia, influências marinhas, continentais e da flutuação do nível do lençol freático (Araujo \& Henriques 1984; Menezes et al. 2010; Magnago et al. 2011).

Por questões como a variação topográfica do relevo, as cristas de cordões arenosos mais elevadas não são influenciadas diretamente pelo lençol freático, condicionando formações vegetais não-inundáveis. Por outro lado, entre cordões ou áreas de depressões, ocorre o afloramento do lençol freático com amplitudes e períodos de inundação variados, condicionando formações vegetais inundáveis e inundadas (Pereira 1990; Araujo 1992; Menezes \& Araujo 2005; Menezes et al. 2010).

$\mathrm{O}$ afloramento do lençol freático pode se dar em diferentes condições, algumas em constante período de inundação perdurando a maior parte do ano, permanecendo o solo encharcado nos períodos de seca, denominando essas formações de inundadas. Onde o lençol é aflorante durante curtos períodos na época chuvosa, as formações são denominadas inundáveis. Esta denominação também pode ser expandida para as formações onde o lençol freático se encontra próximo à superfície do solo, porém, sem afloramento (Menezes \& Araujo 2005). As variações topográficas e o nível do lençol freático podem influenciar a diversidade, composição florística e estrutura da vegetação, constituindo um mosaico de formações vegetais desde herbáceas, tornando-se arbustivas, até arbóreas (Menezes \& Araujo 2005).

As formações arbustivas de restinga se caracterizam pela presença de arbustos tortuosos, formando uma paisagem densa devido à proximidade entre as copas, até moitas de diferentes tamanhos separadas por porções de areia desnuda ou com vegetação herbácea, podendo ser divididas segundo seus atributos fitofisionômicos em fechada e aberta, respectivamente (Henriques et al. 1986; Pereira 1990; Araujo 1992).
No Brasil, principalmente nas regiões sul e sudeste, as restingas têm sido alvo de diversos estudos, sobretudo levantamentos florísticos e estruturais (Araujo \& Henriques 1984; Pereira et al. 1998; Afonso et al. 2007; Menezes et al. 2010; Magnago et al. 2011) que evidenciam a importância desse ecossistema para a biodiversidade (Menezes \& Araujo 2005). O estado do Espírito Santo, com $400 \mathrm{~km}$ de costa, possui cerca de $15 \%$ de vegetação de restinga remanescente (Lani et al. 2008). A maior faixa de planície arenosa situa-se no norte do estado, associada à foz do rio Doce, se estendendo por cerca de $130 \mathrm{~km}$ e apresentando largura máxima de $38 \mathrm{~km}$ (Suguio et al. 1982). Nesta região ocorrem terraços arenosos construídos tanto no Pleistoceno, mais antigo, quanto no Holoceno, mais recente, devido a processos marinhos transgressivos e regressivos, os quais foram responsáveis pela formação de cristas de cordões arenosos e depressões (Martin et al. 1997).

Embora a vegetação de restinga seja foco de estudos recentes ao longo do litoral brasileiro (Amaral et al. 2008; Menezes et al. 2010; Magnago et al. 2011) ainda existe uma defasagem para o Estado do Espírito Santo, principalmente para a região norte, onde os trabalhos que focam a florística das formações vegetais são mais numerosos (Pereira \& Gomes 1993; Behar \& Viégas 1993; Pereira et al. 1998; Colodete \& Pereira 2007) em detrimento daqueles que tratam dos aspectos estruturais (Pereira et al. 2004; Pereira \& Assis 2004). Sendo assim, este trabalho teve como objetivo analisar a estrutura e a composição florística de uma formação de restinga arbustiva aberta no Parque Estadual de Itaúnas, norte do Espírito Santo e verificar as relações florísticas com outras formações similares da região sudeste do Brasil.

\section{Material e Métodos}

Área de estudo

O estudo foi desenvolvido dentro dos limites do Parque Estadual de Itaúnas (PEI) em uma formação arbustiva aberta de restinga localizada na Trilha das Borboletas (18 $\left.24^{\prime} 02^{\prime \prime} \mathrm{S}-39^{\circ} 42^{\prime} 00^{\prime \prime} \mathrm{W}\right)$. O depósito arenoso da área de estudo dista cerca de $1 \mathrm{~km}$ da linha de costa, possui altura em torno de 6 $\mathrm{m}$ acima do nível do mar e é cercado por uma área de depressão onde se desenvolve uma vegetação alagada pelo rio Itaúnas.

A formação vegetal analisada está estabelecida sobre depósitos arenosos construídos devido a processos marinhos transgressivos e 
regressivos que ocorreram durante o Pleistoceno (Martin et al. 1981; Martin et al. 1997). Nesses depósitos arenosos observam-se algumas áreas com afloramento do lençol freático e outras onde, apesar de não aflorar, o lençol freático pode chegar próximo à superfície, inundando a zona das raízes. Desta forma, esta formação foi designada como arbustiva aberta inundável.

Os dados climatológicos foram cedidos pelo Incaper (Instituto Capixaba de Pesquisa, Assistência Técnica e Extensão Rural), a partir da estação do município de São Mateus, a $37 \mathrm{~km}$ da área de estudo. As temperaturas médias anuais registradas para a região foram máximas de $26,7^{\circ} \mathrm{C}$ e mínimas de $21,7^{\circ} \mathrm{C}$, com umidade relativa de $83 \%$ e precipitação média anual de $1308 \mathrm{~mm}$. Pela classificação de Köppen, a região está enquadrada no tipo Aw, portanto, um clima tropical sazonal com as maiores taxas de precipitação nos meses de verão e as menores nos meses de inverno.

A formação estudada é caracterizada por moitas adensadas com 3 a 20 m de diâmetro, distando em torno de 2 a $3 \mathrm{~m}$ umas das outras, apresentando sua porção central ocupada por espécies arborescentes com altura entre 3 e $5 \mathrm{~m}$ e o bordo ocupado, principalmente, por Aechmea blanchetiana e Vriesea neoglutinosa (Bromeliaceae). Na região entre moitas, a vegetação é esparsa e com baixa cobertura, destacando-se Marcetia taxifolia, Paepalanthus klotzschianus e Gomphrena agrestis. Frequentemente, sobre a areia desnuda, Allagoptera arenaria pode se estabelecer e constituir moitas monoespecífica ou associada com Pilosocereus arrabidae.

Estudo e amostragem da comunidade

Para efeito deste estudo foi definido "moita" como sendo um conjunto com mais de dois indivíduos, podendo ocorrer sobreposição de copas, de diâmetro maior ou igual a 0,5 m (Ribas et al. 1993). Essa classificação já foi adotada em outros estudos do gênero (Menezes \& Araujo 2000; Zaluar \& Scarano 2000). Partindo dessa definição, a classificação da vegetação foi realizada por meio de observações de campo sobre aspectos fitofisionômicos, composição florística, forma biológica, abundância de espécies, densidade e profundidade do lençol freático. Foi utilizada nesta classificação a terminologia proposta por Menezes \& Araujo (2005).

Para o levantamento da estrutura da vegetação foi utilizado o método de intercepto de linha (Mueller-Dombois \& Ellenberg 1974). Com o auxílio de uma bússola foram demarcadas 20 linhas por toda extensão da área com $50 \mathrm{~m}$ de comprimento cada, com uma distância de $30 \mathrm{~m}$ uma das outras, totalizando $1000 \mathrm{~m}$ de linha amostrados. Os indivíduos lenhosos e herbáceos com altura igual ou superior a $50 \mathrm{~cm}$ que interceptaram as linhas, exceto lianas, foram incluídos na amostragem. Para cada um destes foram tomadas as seguintes medidas: a extensão do indivíduo interceptado pela projeção da linha e o maior diâmetro de copa perpendicular à linha. Pequenas interrupções de até $5 \mathrm{~cm}$ nas copas dos arbustos interceptados foram ignoradas. Foram coletadas através de caminhada aleatória e incluídas na listagem florística, espécies não contempladas pela amostragem, mas que obedeciam ao critério de inclusão adotado. Foram distribuídos dez pontos entre as unidades amostrais para mensurar a profundidade do lençol freático.

As identificações botânicas foram realizadas através de literatura especializada, comparações com exsicatas já identificadas existentes nos Herbário VIES e CVRD e através de consultas a especialistas. O material botânico fértil foi depositado no Herbário VIES. A classificação de famílias segue o sistema da APG III (2009) para angiospermas e para licófitas e monilófitas foi adotado o proposto por Smith et al. (2006).

\section{Análise estrutural}

Os parâmetros fitossociológicos foram calculados de acordo com Brower \& Zar (1984). Os índices de Densidade Linear e Cobertura Linear são resultantes do número de indivíduos amostrados $(\mathrm{N})$ e intercepto total (L), respectivamente, divididos pelo tamanho da amostragem $(1000 \mathrm{~m})$, sendo seus valores proporcionais e, portanto, desnecessários e suprimidos. O parâmetro de Frequência Absoluta (F) está relacionado à porcentagem de ocorrência da espécie na unidade amostral e o Valor de Importância (VI) é resultante da somatória dos valores relativos de Densidade Linear, Cobertura Linear e Frequência. Optou-se pelo índice de Shannon (H') para o cálculo da diversidade e pelo índice de Pielou $(\mathrm{J})$ para equidade.

\section{Similaridade florística}

Para a análise de similaridade florística utilizou-se o método de ligação UPGMA (Unweighted Pair Group Method using Arithmetic Mean) com emprego do índice de Sørensen, calculado através do programa MVSP (MultiVariate Statistical Package ) versão 3.1 (Kovach 2005), 
com intuito de investigar a relação entre a flora das formações arbustivas abertas de restinga da região sudeste. Foi confeccionado a partir de 10 estudos florísticos e estruturais do Espírito Santo e Rio de Janeiro (Fig. 1) um banco de dados qualitativo de ocorrência de espécies que obedeciam aos critérios de inclusão de amostragem da vegetação adotados neste estudo. Diferentemente das demais, em duas áreas (Montezuma \& Araujo 2000 e Pereira et al. 2001) foram amostradas somente espécies lenhosas, as quais foram consideradas para análise aquelas espécies que obedeciam o critério mínimo de $50 \mathrm{~cm}$ de altura (Tab. 1). A listagem florística deste estudo foi utilizada como parâmetro de comparação com as outras áreas. As sinonímias foram verificadas na base de informações da Lista de Espécies da Flora do Brasil (<http://floradobrasil.jbrj.gov.br/>) e aquelas espécies com determinações incompletas e que não se encaixavam no critério de inclusão utilizado neste estudo foram descartadas da análise, gerando uma matriz binária composta por 221 espécies e 10 áreas estudadas.

\section{Resultados e Discussão}

\section{Composição florística e estrutura}

No levantamento estrutural foram amostradas 42 espécies, distribuídas em 38 gêneros e 28 famílias. As famílias mais ricas em espécies foram

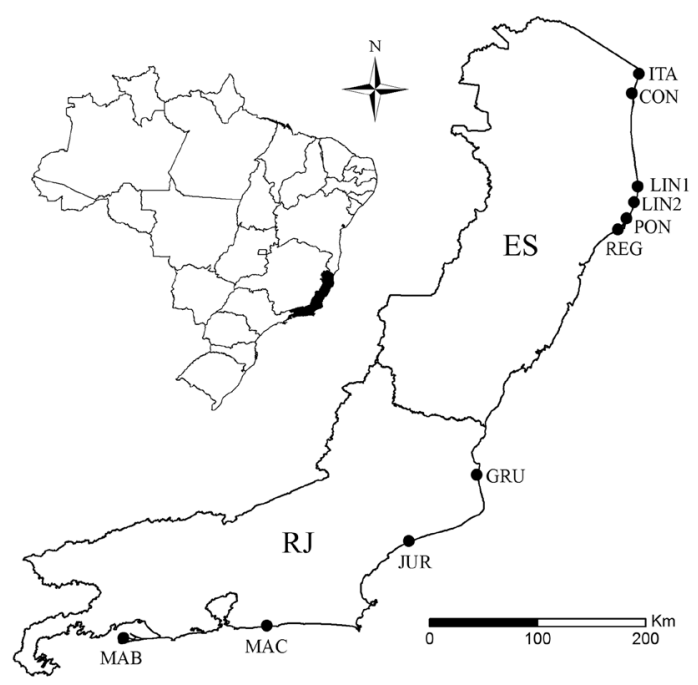

Figura 1 - Localização da área de estudo e distribuição das áreas utilizadas na análise de similaridade florística (abreviaturas das áreas na Tab. 1).

Figure 1 - Location of the study area and distribution of sites used in the floristic similarity analysis (for abbreviations of sites see Tab. 1).
Bromeliaceae e Fabaceae (quatro espécies cada), Apocynaceae, Burseraceae, Clusiaceae, Humiriaceae, Myrsinaceae, Myrtaceae, Orchidaceae e Rubiaceae (duas espécies cada), correspondendo a 57,1\% de todas espécies amostradas. As coletas fora da amostragem corresponderam a 22 espécies adicionadas à listagem florística sendo três espécies de samambaias (pertencentes às famílias Blechnaceae, Polypodiaceae e Schizaeaceae), totalizando 64 espécies (Tab. 2).

Ocorreu uma baixa representatividade de famílias com mais de duas espécies, já que 25 famílias ( $65 \%$ do total) foram representadas na área de estudo por apenas uma espécie, fato observado também em outros estudos em restingas ao longo da costa brasileira (Pereira \& Araujo 2000; Scherer et al. 2005; Amaral et al. 2008). A maioria dos gêneros possui apenas uma espécie (91,5\%), enquanto Aechmea, Clusia, Myrsine, Protium e Tillandsia possuem duas espécies cada. O número de espécies total e por família está próximo ao encontrado em outras comunidades arbustivas abertas e fechadas no Espírito Santo e Rio de Janeiro (Fabris \& Pereira 1998; Assumpção \& Nascimento 2000; Pereira et al. 2001; Pereira \& Assis 2004; Montezuma \& Araujo 2007).

Espécies raras, aquelas representadas por apenas um indivíduo na amostragem (Martins 1991), correspondem a $7,1 \%$ do total amostrado (Tab. 3). Esse valor foi menor em relação a outras comunidades arbustivas contínuas e descontínuas (18\% a 38\%) do Espírito Santo e Rio de Janeiro (Pereira et al. 2001; Pereira \& Assis 2004; Pereira et al. 2004; Montezuma \& Araujo 2007).

Ao todo foram amostrados 1.055 indivíduos, dos quais 463 (44\%) foram não-lenhosos e 592 (56\%) lenhosos. As famílias que compreenderam os indivíduos não-lenhosos foram Bromeliaceae ( $93,2 \%$ do total de indivíduos herbáceos), Arecaceae $(3,7 \%)$, Araceae $(1,4 \%)$, Orchidaceae $(1,2 \%)$ e Cactaceae $(0,5 \%)$. Arecaceae foi classificada desta forma por ser representada apenas por Allagoptera arenaria, uma espécie de caule subterrâneo. As famílias lenhosas com maior número de indivíduos foram Humiriaceae $(15,2 \%$ do total de lenhosas), Burseraceae (13,2\%), Lauraceae $(11,1 \%)$, Myrtaceae $(10 \%)$ e Fabaceae $(6,4 \%)$, perfazendo $31,4 \%$ do total de indivíduos.

$\mathrm{O}$ índice de diversidade encontrado para o trecho em questão foi de $\mathrm{H}^{\prime}=2,83$ nats/ind., enquanto que o valor de $J=0,75$. $O$ índice de diversidade foi o mais baixo registrado em comparação a outras formações arbustivas do Espírito Santo (Pereira \& Assis 2004; Pereira et al. 2004). 
Tabela 1 - Formações arbustivas de restinga do Rio de Janeiro e Espírito Santo utilizadas na análise de similaridade florística: $\mathrm{ABI}=$ Arbustiva aberta inundável; $\mathrm{ABNI}=$ Arbustiva aberta não inundável; $\mathrm{AFI}=$ Arbustiva fechada inundável; AFNI = arbustiva fechada não inundável. PEI = Parque Estadual de Itaúnas; PNRJ = Parque Nacional da Restinga de Jurubatiba. Métodos: F = florística; $\mathrm{I}=$ intercepto de linha; $\mathrm{T}=$ transecção. Hábito: $\mathrm{H}=$ herbáceo; $\mathrm{L}=$ lenhoso.

Table 1 - Information on scrub restinga formations of Rio de Janeiro and Espírito Santo used in floristic similarity. ABI = Flooded open scrub; ABNI = Non-flooded open scrub; AFI = Flooded close scrub; AFNI = Non-flooded close scrub. PEI = Itaúnas State Park; PNRJ $=$ Restinga de Jurubatiba National Park. Methods: $\mathrm{F}=$ floristic; $\mathrm{I}=$ line intercept; $\mathrm{T}=$ transect. Habits: $\mathrm{H}=$ herbaceous; $\mathrm{L}=$ woody.

\begin{tabular}{|c|c|c|c|c|c|c|c|}
\hline \multirow[b]{2}{*}{ Município } & \multicolumn{6}{|c|}{ Formação } & \multirow[b]{2}{*}{ Referência } \\
\hline & UF & Localidade & Abreviatura & vegetal & Método & Hábito & \\
\hline Linhares & ES & Pontal do Ipiranga & PON & ABNI & $\mathrm{F}$ & $\mathrm{He} \mathrm{L}$ & Pereira et al. 1998 \\
\hline Linhares & ES & Regência & REG & ABNI & $\mathrm{F}$ & $\mathrm{He} \mathrm{L}$ & Colodete \& Pereira 2007 \\
\hline Linhares & ES & Sem indicação & LIN1 & ABNI & $\mathrm{I}(640 \mathrm{~m})$ & $\mathrm{He} \mathrm{L}$ & Pereira et al. 2004 \\
\hline Linhares & ES & Sem indicação & LIN2 & AFI & I (640m) & $\mathrm{H}$ e L & Pereira \& Assis 2004 \\
\hline Conceição da Barra & ES & Sem indicação & $\mathrm{CON}$ & AFI & $\mathrm{F}$ & $\mathrm{He} \mathrm{L}$ & Pereira \& Gomes 1994 \\
\hline Conceição da Barra & ES & PEI & ITA & $\mathrm{ABI}$ & $\mathrm{I}(1000 \mathrm{~m})$ & $\mathrm{He} \mathrm{L}$ & Este estudo \\
\hline São João da Barra & RJ & Grussaí & GRU & ABNI & $\mathrm{I}(500 \mathrm{~m})$ & $\mathrm{He} \mathrm{L}$ & Assumpção \& Nascimento 2000 \\
\hline Carapebus & RJ & PNRJ & JUR & $\mathrm{ABI}$ & $\mathrm{T}$ (1ha) & $\mathrm{L}$ & Montezuma \& Araujo 2007 \\
\hline Maricá & RJ & Barra de Maricá & MAC & AFNI & $\mathrm{I}(350 \mathrm{~m})$ & $\mathrm{L}$ & Pereira et al. 2001 \\
\hline Rio de Janeiro & RJ & Marambaia & MAB & ABNI & $\mathrm{I}(1000 \mathrm{~m})$ & $\mathrm{H}$ e L & Afonso et al. 2007 \\
\hline
\end{tabular}

Tabela 2 - Lista florística de uma restinga arbustiva aberta inundável no Parque Estadual de Itaúnas, Conceição da Barra, Espírito Santo (*Espécies incluídas na amostragem). Fbiol = forma biológica. $\mathrm{H}=$ Herbácea; $\mathrm{L}=$ Lenhosa.

Table 2 - Floristic list of a flooded open scrub restinga in Itaúnas State Park, Conceição da Barra, Espírito Santo (* Species included in the sample). Fbiol $=$ life form. $\mathrm{H}=$ Herbaceous; $\mathrm{L}=$ Woody.

\begin{tabular}{llc}
\hline Família & Espécie & Fbiol \\
\hline AMARANTHACEAE & Gomphrena agrestis Mart. & $\mathrm{H}$ \\
ANACARDIACEAE & Anacardium occidentale L.* & $\mathrm{L}$ \\
& Tapirira guianensis Aubl. & $\mathrm{L}$ \\
APOCYNACEAE & Hancornia speciosa Gomes* & $\mathrm{L}$ \\
& Himatanthus phagedaenicus (Mart.) Woodson* & $\mathrm{L}$ \\
AQUIFOLIACEAE & Ilex integerrima (Vell.) Reissek * & $\mathrm{L}$ \\
ARACEAE & Anthurium raimundii Mayo, Haigh \& Nadruz* & $\mathrm{H}$ \\
ARALIACEAE & Schefflera selloi (Marchal) Frodin \& Fiaschi* & $\mathrm{L}$ \\
ARECACEAE & Allagoptera arenaria (Gomes) Kuntze* & $\mathrm{H}$ \\
BLECHNACEAE & Blechnum serrulatum Rich. & $\mathrm{H}$ \\
BROMELIACEAE & Aechmea blanchetiana (Baker) L.B.Sm.* & $\mathrm{H}$ \\
& Aechmea nudicaulis (L.) Griseb.* & $\mathrm{H}$ \\
& Neoregelia cruenta (R.Graham) L.B.Sm.* & $\mathrm{H}$ \\
& Tillandsia stricta Sol. & $\mathrm{H}$ \\
BURSERACEAE & Tillandsia usneoides (L.) L. & $\mathrm{H}$ \\
& Vriesea neoglutinosa Mez* & $\mathrm{H}$ \\
& Protium heptaphyllum (Aubl.) Marchand* & $\mathrm{L}$ \\
\hline
\end{tabular}




\begin{tabular}{|c|c|c|}
\hline Família & Espécie & Fbiol \\
\hline & Protium icicariba (DC.) Marchand* & $\mathrm{L}$ \\
\hline CACTACEAE & Pilosocereus arrabidae (Lem.) Byles \& Rowley* & $\mathrm{H}$ \\
\hline CELASTRACEAE & Maytenus distichophylla Mart. ex Reissek & $\mathrm{L}$ \\
\hline CHRYSOBALANACEAE & Hirtella corymbosa Cham. \& Schltdl.* & $\mathrm{L}$ \\
\hline \multirow[t]{2}{*}{ CLUSIACEAE } & Clusia hilariana Schltdl.* & $\mathrm{L}$ \\
\hline & Clusia spiritu-sanctensis G.Mariz \& B.Weinberg* & $\mathrm{L}$ \\
\hline CYPERACEAE & Lagenocarpus rigidus Nees & $\mathrm{H}$ \\
\hline ERICACEAE & Gaylussacia brasiliensis (Spreng.) Meisn.* & $\mathrm{L}$ \\
\hline \multirow[t]{2}{*}{ ERIOCAULACEAE } & Actinocephalus ramosus (Wikstr.) Sano & $\mathrm{H}$ \\
\hline & Paepalanthus klotzschianus Körn. & $\mathrm{H}$ \\
\hline \multirow[t]{7}{*}{ FABACEAE } & Abarema filamentosa (Benth.) Pittier* & $\mathrm{L}$ \\
\hline & Andira nitida Mart. ex Benth.* & $\mathrm{L}$ \\
\hline & Chamaecrista ensiformis (Vell.) H.S. Irwin \& Barneby & $\mathrm{L}$ \\
\hline & Inga laurina (Sw.) Willd. & $\mathrm{L}$ \\
\hline & Leptolobium bijugum (Spreng.) Voge1* & $\mathrm{L}$ \\
\hline & Stylosanthes scabra Vogel & $\mathrm{H}$ \\
\hline & Swartzia apetala Raddi* & $\mathrm{L}$ \\
\hline \multirow[t]{2}{*}{ HUMIRIACEAE } & Humiria balsamifera (Aubl.) J.St.-Hil.* & $\mathrm{L}$ \\
\hline & Vantanea bahiaensis Cuatrec.* & $\mathrm{L}$ \\
\hline ICACINACEAE & Emmotum nitens (Benth.) Miers* & $\mathrm{L}$ \\
\hline LAURACEAE & Ocotea notata (Nees \& Mart.) Mez* & $\mathrm{L}$ \\
\hline MALPIGHIACEAE & Byrsonima sericea DC.* & $\mathrm{L}$ \\
\hline \multirow[t]{3}{*}{ MELASTOMATACEAE } & Marcetia taxifolia (A. St.-Hil.) DC.* & $\mathrm{L}$ \\
\hline & Miconia cinnamomifolia (DC.) Naudin & $\mathrm{L}$ \\
\hline & Tibouchina urceolaris (Schrank \& Mart. ex DC.) Cogn. & $\mathrm{L}$ \\
\hline MORACEAE & Ficus cf. bahiensis C.C.Berg \& Carauta* & $\mathrm{L}$ \\
\hline \multirow[t]{5}{*}{ MYRTACEAE } & Calyptranthes brasiliensis Spreng.* & $\mathrm{L}$ \\
\hline & Marlierea neuwiedeana (O. Berg) Nied.* & $\mathrm{L}$ \\
\hline & Myrciaria floribunda (H. West ex Willd.) O.Berg & $\mathrm{L}$ \\
\hline & Neomitranthes obtusa Sobral \& Zambom & $\mathrm{L}$ \\
\hline & Psidium brownianum DC. & $\mathrm{L}$ \\
\hline NYCTAGINACEAE & Guapira opposita (Vell.) Reitz* & $\mathrm{L}$ \\
\hline OCHNACEAE & Ouratea cuspidata (A.St.-Hil.) Engl.* & $\mathrm{L}$ \\
\hline \multirow[t]{2}{*}{ ORCHIDACEAE } & Epidendrum denticulatum Barb. Rodr.* & $\mathrm{H}$ \\
\hline & Koellensteinia altissima Pabst* & $\mathrm{H}$ \\
\hline PERACEAE & Pera heteranthera (Schrank) I.M.Johnst. & $\mathrm{L}$ \\
\hline POLYGALACEAE & Securidaca lanceolata A.St.-Hil. \& Moq. & $\mathrm{L}$ \\
\hline POLYPODIACEAE & Serpocaulon triseriale (Sw.) A.R.Sm.* & $\mathrm{H}$ \\
\hline
\end{tabular}




\begin{tabular}{llc}
\hline Família & Espécie & Fbiol \\
\hline PRIMULACEAE & Myrsine parvifolia A. DC.* & $\mathrm{L}$ \\
& Myrsine umbellata Mart.* & $\mathrm{L}$ \\
RUBIACEAE & Chiococca nitida Benth.* & $\mathrm{L}$ \\
& Pagamea cf. guianensis Aubl.* & $\mathrm{L}$ \\
RUTACEAE & Conchocarpus insignis Pirani & $\mathrm{L}$ \\
SAPOTACEAE & Manilkara subsericea (Mart.) Dubard* & $\mathrm{L}$ \\
SCHIZAEACEAE & Actinostachys pennula (Sw.) Hook. & $\mathrm{H}$ \\
SHOEPFIACEAE & Schoepfia brasiliensis A.DC.* & $\mathrm{L}$ \\
SOLANACEAE & Solanum thomasiifolium Sendtn. & $\mathrm{L}$ \\
\hline
\end{tabular}

Tabela 3 - Espécies amostradas em uma restinga arbustiva aberta inundável no Parque Estadual de Itaúnas e seus parâmetros fitossociológicos. $\mathrm{N}$ = número total de indivíduos; $\mathrm{F}=$ frequência absoluta; $\mathrm{L}=$ intercepto total; $\mathrm{VI}=$ valor de importância. O número de intervalos de ocorrências das espécies foi o mesmo que o $\mathrm{N}$, exceto para Aechmea blanchetiana $=57$, Vriesea neoglutinos $a=79$, Neoregelia cruenta $=6$ e Serpocaulon triseriale $=3$.

Table 3 - Species sampled on a flooded open scrub restinga in Itaúnas State Park and its phytosociological parameters. N= total number of individuals; $\mathrm{F}=$ absolute frequency; $\mathrm{L}=$ total intercept; $\mathrm{VI}=$ importance value. The number of intervals of the species occurrence was the same as N, except for Aechmea blanchetiana $=57$, Vriesea neoglutinosa $=79$, Neoregelia cruenta $=6$ e Serpocaulon triseriale $=3$.

\begin{tabular}{clcccc}
\hline & Espécie & $\mathbf{N}$ & $\mathbf{F}$ & $\mathbf{L}(\mathbf{m})$ & $\mathbf{V I}$ \\
\hline 1 & Aechmea blanchetiana & 290 & 15 & 112,42 & 42,58 \\
2 & Humiria balsamifera & 80 & 19 & 171,87 & 29,35 \\
3 & Protium heptaphyllum & 59 & 18 & 130,50 & 23,31 \\
4 & Vriesea neoglutinosa & 134 & 18 & 48,06 & 22,99 \\
5 & Ocotea notata & 66 & 18 & 91,72 & 20,48 \\
6 & Marlierea neuwiedeana & 43 & 16 & 45,96 & 13,51 \\
7 & Clusia hilariana & 23 & 16 & 61,17 & 12,99 \\
8 & Myrsine umbellata & 37 & 16 & 33,15 & 11,79 \\
9 & Emmotum nitens & 17 & 10 & 70,96 & 11,31 \\
10 & Hirtella corymbosa & 35 & 14 & 29,15 & 10,58 \\
11 & Gaylussacia brasiliensis & 32 & 12 & 18,31 & 8,66 \\
12 & Schoepfia bahiensis & 23 & 11 & 25,81 & 8,15 \\
13 & Protium icicariba & 19 & 6 & 32,85 & 6,75 \\
14 & Vantanea bahiensis & 10 & 5 & 44,90 & 6,65 \\
15 & Calyptranthes brasiliensis & 16 & 9 & 18,33 & 6,15 \\
16 & Allagoptera arenaria & 17 & 8 & 14,49 & 5,57 \\
17 & Andira nitida & 16 & 7 & 16,63 & 5,33 \\
18 & Leptolobium bijugum & 9 & 7 & 21,61 & 5,12 \\
19 & Marcetia taxifolia & 17 & 8 & 9,44 & 5,11 \\
20 & Ilex integerrima & 14 & 8 & 10,16 & 4,89 \\
21 & Swartzia apetala & 10 & 8 & 5,26 & 4,07 \\
22 & Chiococca nitida & 10 & 5 & 15,49 & 4,00 \\
\hline
\end{tabular}




\begin{tabular}{llcccc}
\hline & Espécie & $\mathbf{N}$ & $\mathbf{F}$ & $\mathbf{L}(\mathbf{m})$ & $\mathbf{V I}$ \\
\hline 23 & Schefflera selloi & 8 & 6 & 10,77 & 3,71 \\
24 & Clusia spiritu-sanctensis & 7 & 4 & 11,24 & 3,00 \\
25 & Anthurium raimundii & 7 & 6 & 1,54 & 2,79 \\
26 & Neoregelia cruenta & 11 & 4 & 3,79 & 2,71 \\
27 & Ficus cf. bahiensis & 3 & 3 & 13,90 & 2,53 \\
28 & Ouratea cuspidata & 7 & 4 & 3,46 & 2,30 \\
29 & Abarema filamentosa & 3 & 2 & 8,45 & 1,71 \\
30 & Byrsonima sericea & 2 & 2 & 6,62 & 1,45 \\
31 & Serpocaulon triseriale & 6 & 2 & 2,25 & 1,43 \\
32 & Manilkara subsericea & 5 & 2 & 2,86 & 1,39 \\
33 & Anacardium occidentale & 2 & 1 & 5,85 & 1,05 \\
34 & Guapira opposita & 3 & 2 & 0,97 & 1,03 \\
35 & Epidendrum denticulatum & 2 & 2 & 2,00 & 1,03 \\
36 & Pagamea guianensis & 2 & 2 & 1,85 & 1,02 \\
37 & Himatanthus phagedaenicus & 2 & 1 & 2,60 & 0,75 \\
38 & Koellensteinia altissima & 3 & 1 & 1,20 & 0,72 \\
39 & Pilosocereus arrabidae & 2 & 1 & 0,70 & 0,58 \\
40 & Hancornia speciosa & 1 & 1 & 1,00 & 0,52 \\
41 & Aechmea nudicaulis & 1 & 1 & 0,75 & 0,49 \\
42 & Myrsine parvifolia & 1 & 1 & 0,35 & 0,46 \\
\hline & Total & 1055 & 302 & 1110 & 300 \\
\hline
\end{tabular}

Os valores do índice de diversidade e de equidade foram ainda mais baixos no estudo de Montezuma \& Araujo (2007) $\left(\mathrm{H}^{\prime}=2,63\right.$ e J $=$ $0,70)$ do que no presente estudo. Esse baixo valor do índice de diversidade pode estar refletindo o elevado grau de seletividade de espécies nesse ambiente e uma estrutura oligárquica, onde poucas espécies contribuem com a maior parte da dominância. Isso fica mais evidente quando são eliminadas da amostragem as espécies herbáceas, perfazendo 34 espécies lenhosas e índice de diversidade $H^{\prime}=3,05$ e $\mathbf{J}=0,86$. Dessa maneira, a inclusão metodológica do estrato herbáceo demonstrou o processo de hierarquização das espécies no ambiente estudado, o que contribuiu para o decréscimo do índice de diversidade.

As formações arbustivas abertas variam quanto à cobertura vegetal total, onde, em Jurubatiba (RJ), foi encontrado uma cobertura vegetal entre $35 \%$ e $44 \%$ (Araujo et al. 2004; Montezuma \& Araujo 2007), enquanto que em Maricá (RJ) a cobertura vegetal compreendeu
$90 \%$ da formação (Pereira et al. 2001). Em Itaúnas foi observado uma cobertura vegetal total de $70 \%$. Essa variação da cobertura pode estar relacionada a fatores ambientais como variação do lençol freático e salinidade (Magnago et al. 2011).

Neste estudo, as 10 espécies mais importantes representaram $65,6 \%$ do VI total. Verificou-se que em duas comunidades arbustivas descontínuas em Linhares (ES), as 10 e 14 espécies melhores colocadas representaram cerca de $75 \%$ do VI total (Pereira \& Assis 2004 e Pereira et al. 2004, respectivamente). As cinco famílias mais importantes correspondem a $58 \%$ do VI total. A maior porcentagem do VI total de cada família foi ocupada por apenas uma espécie, sendo Aechmea blanchetiana com $62 \%$ do VI total da família, Humiria balsamifera com 81,5\%, Protium heptaphyllum com $77,6 \%$, Ocotea notata com $100 \%$ e Marlierea neuwiedeana com $68,8 \%$. Isso fica ainda mais evidente ao analisar o estrato herbáceo, onde A. blanchetiana e Vriesea neoglutinosa juntas compreendem $70 \%$ do total 
de indivíduos herbáceos. Em geral, formações de baixa riqueza de espécies possuem uma estrutura mantida por um número ainda menor de espécies, válido tanto no estrato lenhoso quanto no herbáceo, podendo isso refletir a influência de diversos fatores que exercem forte pressão seletiva (Sá \& Araujo 2009; Carvalho \& Sá 2011; Magnago et al. 2011).

Bromeliaceae está entre as famílias mais ricas nas restingas do Rio de Janeiro e do Espírito Santo (Pereira \& Araujo 2000), sendo que neste estudo representou apenas cerca de $7 \%$ do total de espécies. Entretanto, essa família compreendeu cerca de $40 \%$ do total de indivíduos. As espécies de Bromeliaceae desempenham um importante papel na estrutura da comunidade devido a sua capacidade adaptativa em conquistar habitats diversos (Henriques et al. 1986; Martinelli 2006). Avaliando a estrutura da comunidade de Bromeliaceae na restinga de Jurubatiba (RJ), Cogliatti-Carvalho et al. (2001) verificaram que a formação com maior densidade dessa família foi a restinga aberta de Ericaceae que, embora não seja equivalente à formação em questão, sofre influência direta do lençol freático, (Henriques et al. 1986). A proximidade do lençol freático à superfície desempenha um importante papel na fisionomia da comunidade (Montezuma \& Araujo 2007), podendo explicar neste estudo a elevada densidade de Aechmea blanchetiana e Vriesea neoglutinosa que ocuparam a primeira e quarta posição, respectivamente, em ordem decrescente de VI. Essas espécies juntas corresponderam a $95,4 \%$ do VI total da família. A primeira espécie geralmente se distribuiu ao longo da borda da moita, mas pode ocupar também seu interior, algumas vezes encobrindo quase todo o solo, desta maneira, possuindo a terceira maior cobertura.

Montezuma \& Araujo (2007), avaliando o estrato lenhoso na restinga de Jurubatiba (RJ) de uma formação arbustiva inundável, observaram que Humiria balsamifera se destacou pela elevada dominância e Protium icicariba e Ocotea notata pela alta densidade, sendo as espécies que tiveram as maiores expressões estruturais. De maneira semelhante, neste estudo $H$. balsamifera, $P$. heptaphyllum e O. notata foram as lenhosas melhores colocadas segundo o VI, com elevada dominância e frequência, podendo isso ser atribuído às semelhantes características ambientalmente limitantes, como a proximidade do lençol freático com a superfície, podendo assim serem apontadas como espécies que caracterizam esta formação.
Com medições do nível do lençol freático abaixo da superfície foi possível identificar uma variação da profundidade de 0,67 a 1,20 m distribuídos ao longo da área de estudo, onde áreas de nível topográfico distintos determinam o solo mais ou menos úmido, podendo isto influenciar na estrutura da vegetação (Montezuma \& Araujo 2007) e funcionar como fator seletivo de espécies (Magnago et al. 2011). A proximidade do lençol freático com a superfície, inundando a zona das raízes e exercendo influência no processo de estabelecimento e desenvolvimento das espécies (Scarano 1998) se mostra um dos principais fatores abióticos responsáveis pela variação intra e/ou interespecífica (Duarte et al. 2005).

\section{Similaridade florística}

$O$ coeficiente de similaridade entre as restingas analisadas variou entre 0,07 e 0,48 , revelando poucas semelhanças florísticas entre estas, já que valores menores que 0,5 exprimem baixa similaridade (Kent \& Coker 1992). O maior valor do coeficiente de similaridade registrado com o presente estudo (ITA) foi com um estudo em Conceição da Barra (CON), enquanto que o menor valor do coeficiente foi registrado com a restinga de Marambaia (MAB) $(0,33$ e 0,16, respectivamente). Além disso, quando comparado os valores de similaridade entre o presente estudo (ITA) e os estudos desenvolvidos no Espírito Santo (CON, LIN, PON, REG), todos na região norte, observase que, apesar de representarem os maiores valores de similaridade registrados com o presente estudo, ainda são considerados baixos. Sendo assim, a heterogeneidade florística evidenciada pela análise de agrupamento demonstrou que, mesmo sendo geograficamente próximas, as formações arbustivas de restinga do Espírito Santo e Rio de Janeiro possuem características peculiares a cada região como resultado de interações abióticas e bióticas (Magnago et al. 2011), como as pequenas variações na elevação dos cordões arenosos, controlando o regime hídrico de saturação do solo (Menezes et al. 2010) ou a influência de ecossistemas adjacentes sobre a composição de espécies (Giaretta et al. 2013). Para alcançar tais conclusões é necessário cuidado, já que as diferenças florísticas entre os estudos comparados podem ser reflexo dos critérios metodológicos adotados. Contudo, isso não invalida o padrão geral de que as restingas do Espírito Santo e Rio de Janeiro formaram dois blocos distintos pelas suas diferenças florísticas (Fig. 2). 


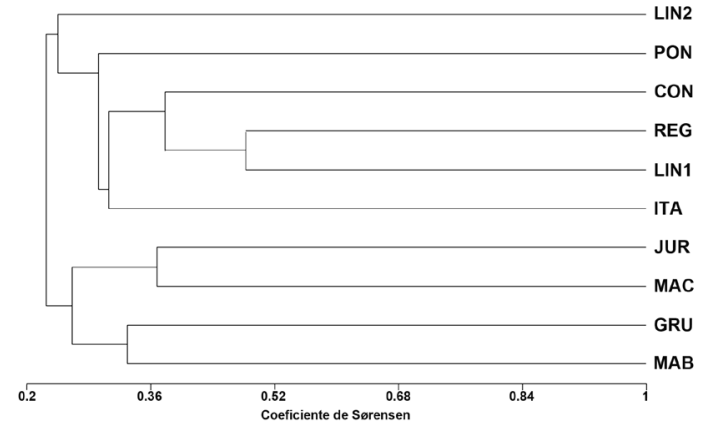

Figura 2 - Dendrograma de similaridade entre formações arbustivas de restinga do sudeste do Brasil, usando uma matriz binária de presença/ausência, o índice de Sørensen e ligação média entre grupos (UPGMA).

Figure 2 - Similarity dendrogram (UPGMA) between restinga scrub formations in southeastern Brazil, using a binary matrix of presence/absence, the Sørensen index and the average linkage between groups (UPGMA).

A distância entre florestas vizinhas é um fator relevante que determina variações graduais na composição florística da restinga (Scherer et al. 2005), assim, os blocos formados na análise podem refletir o distanciamento entre as áreas analisadas. Entretanto, Manilkara subsericea, Pilosocereus arrabidae e Byrsonima sericea foram comuns a todas as restingas analisadas, portanto, espécies que podem ser consideradas de ampla distribuição ao longo da costa sudeste, destacando-se esta última por ser a única registrada em todos os estudos analisados.

O dendrograma de similaridade revelou que as formações arbustivas analisadas não se agruparam exclusivamente pelas condições de saturação hídrica do solo (formação inundável ou não inundável) ou pela fitofisionomia (formação aberta ou fechada). O coeficiente de similaridade entre as restingas de Regência (REG) e Linhares (LIN1) foi o maior registrado nesta análise $(0,48)$, sendo que a explicação disto resulta da proximidade entre as áreas e por ambas se tratarem de formações de restinga arbustiva abertas não inundáveis. As outras áreas analisadas do Espírito Santo (CON, ITA, LIN2 e PON) se mostraram pouco similares floristicamente. Essas formações, exceto PON, são inundáveis e influenciadas diretamente pelas flutuações do lençol freático, portanto, reconhecidamente com baixa riqueza (Magnago et al. 2011), reduzindo a possibilidade de compartilhamento de espécies com outras restingas não inundáveis.
As formações de restinga do Rio de Janeiro formaram um grupo coeso $(0,38)$, onde Jurubatiba (JUR) e Maricá (MAC), a primeira arbustiva aberta inundável e a segunda arbustiva fechada não inundável, se mostraram mais similares floristicamente do que Grussaí (GRU) e Marambaia (MAB), ambas formações arbustiva aberta não inundáveis $(0,33)$. Dessa maneira, os dados sugerem que o aspecto fitofisionômico da formação de restinga arbustiva (aberta ou fechada) pode ser mais relevante para determinar a similaridade na composição de espécies do que a proximidade entre as áreas. Entretanto, essa conclusão deve ser feita com cautela já que em JUR e MAC o estrato herbáceo não foi contemplado pela amostragem, o que pode ter influenciado nos resultados da análise reduzindo o grau de dissimilaridade entre as áreas.

A forte estrutura oligárquica da comunidade estudada foi visível tanto no estrato lenhoso quanto no herbáceo, mostrando que as condições limitantes do ambiente da restinga foram muito importantes para o baixo índice de diversidade encontrado. Os resultados da similaridade florística evidenciaram que as formações arbustivas do Rio de janeiro e Espírito Santo formaram dois blocos florísticos distintos. A importância da conservação desta formação arbustiva aberta em Itaúnas reside na manutenção dos processos ecológicos e no entendimento de como a variação nos aspectos fisionômicos e ambientais das formações de restinga arbustiva da costa sudeste influenciam no estabelecimento das espécies.

\section{Agradecimentos}

Ao Parque Estadual de Itaúnas pelo apoio logístico durante todo o desenvolvimento desta pesquisa; ao Conselho Nacional de Desenvolvimento Científico e Tecnológico (CNPq) pela concessão de bolsa ao primeiro e segundo autores; à FAPES por patrocinar parte deste estudo; aos diversos especialistas pelas identificações das espécies.

\section{Referências}

Afonso, A.S.; Medeiros, A.S.; Nunes, C.S.; Rodrigues, G.A.; Nunes, R.S.; Menezes, L.F.T. \& Conde, M.M.S. 2007. Florística da vegetação arbustiva aberta na Restinga da Marambaia, RJ. Revista Brasileira de Biociências 5: 450-452.

Amaral, D.D.; Prost, M.T.; Bastos, M.N.C.; Neto, S.V.C. \& Santos, J.U.M. 2008. Restingas do litoral amazônico, estados do Pará e Amapá, Brasil. Boletim do Museu Paraense Emílio Goeldi, Ciências Naturais 3: 35-67. 
Angiosperm Phylogeny Group III (APG III). 2009. An update of the Angiosperm Phylogeny Group classification for the orders and families of flowering plants: APG III. Botanical Journal of the Linnean Society 161: 105-121.

Araujo, D.S.D. \& Henriques, R.P.B. 1984. Análise florística das restingas do estado do Rio de Janeiro. In: Lacerda, L.D.; Araujo, D.S.D.; Cerqueira, R. \& Turc, B. (orgs.). Restingas: origem, estrutura e processos. CEUFF, Niterói. Pp. 150-193.

Araujo, D.S.D. 1992. Vegetation types of sandy coastal plains of tropical Brazil: a first approximation. In: Seeliger, U. (ed.). Coastal plant communities of Latin America. Academic Press, New York, Pp. 337-347.

Araujo, D.S.D.; Pereira, M.C.A. \& Pimentel, M.C.P. 2004. Flora e estrutura de comunidades na Restinga de Jurubatiba - síntese dos conhecimentos com enfoque especial para a formação aberta de Clusia. In: Rocha, C.F.D.; Esteves, F.A. \& Scarano, F.R. (eds.). Pesquisas de longa duração na Restinga de Jurubatiba: ecologia, história natural e conservação. RiMa, São Carlos. Pp. 59-76.

Assumpção, J. \& Nascimento, M.T. 2000. Estrutura e composição florística de quatro formações vegetais de restinga no complexo lagunar Grussaí/Iquipari, São João da Barra, RJ, Brasil. Acta Botanica Brasilica 14: 301-315.

Behar, L. \& Viégas, G.M.S. 1993. Pteridophyta da Restinga de Comboios, Aracruz/Linhares-ES. In: Anais II Simpósio de Ecossistemas da Costa Brasileira. ACIESP, São Paulo. Pp. 34-43.

Brower, J.E. \& Zar, J.H. 1984. Field \& laboratory methods for general ecology. $2^{\text {nd }}$ ed. Brown Publishers, Boston. 226p.

Carvalho, D. A. \& Sá, C.F.C. 2011. Estrutura do estrato herbáceo de uma restinga arbustiva aberta na APA de Massambaba, Rio de Janeiro, Brasil. Rodriguésia 62: 367-378.

Cogliatti-Carvalho, L.; Nunes-Freitas, A.F.; Rocha, C.F.D. \& Van Sluys, M. 2001.Variação na estrutura e composição de Bromeliaceae em cinco zonas de vegetação no Parque Nacional da Restinga de Jurubatiba, Macaé, RJ. Revista Brasileira de Botânica 24:1-9.

Colodete, M.F. \& Pereira, O.J. 2007. Levantamento florístico da restinga de Regência, Linhares, ES. Revista Brasileira de Biociências 5: 558-560.

Duarte, H.M.; Gebler, A.; Scarano, F.R.; Franco, A.C.; Mattos, E.A.; Nahm, M.; Rennenberg, H.; Rodrigues, P.J.F.P.; Zaluar, H.L.T. \& Lüttge, U. 2005. Ecophysiology of six selected shrub species in different plant communities at the periphery of the Atlantic Forest of SE, Brazil. Flora 200: 456-476.

Fabris, L.C. \& Pereira, O.J. 1998. Florística da formação pós-praia, na restinga de Setiba, município de Guarapari (ES). In: Anais do IV Simpósio de Ecossistemas Brasileiros. Vol. 3. ACIESP, São Paulo. Pp. 165-176.
Giaretta, A.; Menezes, L.F.T. \& Pereira, O.J. 2013. Structure and floristic pattern of a restinga in southeastern Brazil. Acta Botanica Brasilica 27: 87-107.

Henriques, R.P.B.; Araujo, D.S.D. \& Hay, J.D. 1986. Descrição e classificação dos tipos de vegetação da restinga de Carapebus, Rio de Janeiro. Revista Brasileira de Botânica 9: 173-189.

Kent, M. \& Coker, P. 1992. Vegetation description and analysis: a practical approach. Belhaven Press, London. 363p.

Kovach, W. L. 2005. MVSP - A MultiVariate Statistical Package for Windows, ver. 3.1. Kovach Computing Services, Pentraeth.

Lani, J.L.; Resende, M.; Rezende, S.B. \& Feitoza, L.R. (eds.). 2008. Atlas de ecossistemas do Espírito Santo. GSA, SEMA, Viçosa. 504p.

Lista de Espécies da Flora do Brasil. Disponível em $<$ http://floradobrasil.jbrj.gov.br/>. Acesso em 19 Abr 2013.

Magnago, L.F.S.; Pereira, O.J. \& Martins, S.V. 2011. Caracterização das formações ribeirinhas na restinga do Parque Natural Municipal de Jacarenema, Vila Velha, ES, Brasil. In: Felfili, J.M.; Eisenlohr, P.V.; Melo, M.M.R.F.; Andrade, L.A. \& Meira-Neto, J.A.A. (eds.). Fitossociologia do Brasil: métodos e estudos de caso. Vol. 1. Ed. UFV, Viçosa. Pp. 415-440.

Martin, L.; Bittencourt, A.C.S.P. \& Vilas-Boas, G.S. 1981. Différenciation sur photographies aériennes des terrasses sableuses marines pléistocènes et holocènes du littoral de l'état de Bahia (Brésil). Photointerprétation 20: 1-10.

Martin, L.; Suguio, K.; Dominguez, J.M.L. \& Flexor, J.M. 1997. Geologia do quaternário costeiro do litoral norte do Rio de Janeiro e do Espírito Santo. CPRM/FAPESP, Belo Horizonte. 112p.

Martinelli, G. 2006. Manejo de populações e comunidades vegetais: um estudo de caso na conservação de Bromeliaceae. In: Rocha, C.F.D.; Bergallo, H.G.; Van Sluys, M. \& Alves, M.A.S. (orgs.). Biologia da conservação: essências. RiMa, São Carlos. Pp. 479-503.

Martins, F.R. 1991. Estrutura de uma floresta mesófila. Série Teses. Ed. da Universidade Estadual de Campinas, Campinas. 246 p.

Menezes, L.F.T. \& Araujo, D.S.D. 2000. Variação da biomassa aérea de Allagoptera arenaria (Gomes) O. Kuntze (Arecaceae) em uma comunidade arbustiva de Palmae na Restinga da Marambaia, RJ. Revista Brasileira de Biologia 60: 147-157.

Menezes, L.F.T. \& Araujo, D.S.D. 2005. Formações vegetais da Restinga da Marambaia. In: Menezes, L.F.T.; Peixoto; A.L. \& Araujo, D.S.D. (orgs.). História natural da Marambaia. Seropédica, Ed. da Universidade Federal Rural do Rio de Janeiro. Pp. 67-120. 
Menezes, L.F.T.; Araujo, D.S.D. \& Nettesheim, F.C. 2010. Estrutura comunitária e amplitude ecológica do componente lenhoso de uma floresta de restinga mal drenada no Sudeste do Brasil. Acta Botanica Brasilica 24: 825-839.

Montezuma, R.C.M. \& Araujo, D.S.D. 2007. Estrutura da vegetação de uma restinga arbustiva inundável no Parque Nacional da Restinga de Jurubatiba, Rio de Janeiro. Pesquisas Botânica, São Leopoldo: Instituto Anchietano de Pesquisa 58: 157-176.

Mueller-Dombois, D. \& Ellenberg, H. 1974. Aims and methods of vegetation ecology. John Wiley \& Sons, New York. 547p.

Pereira, M.C.A.; Araujo, D.S.D. \& Pereira, O.J. 2001. Estrutura de uma comunidade arbustiva da restinga de Barra de Maricá - RJ. Revista Brasileira de Botânica 24: 273-281.

Pereira, O.J. \& Araujo, D.S.D. 2000. Análise florística das restingas dos estados do Espírito Santo e Rio de Janeiro. In: Esteves, F.A. \& Lacerda, L.D. (orgs.). Ecologia de restingas e lagoas costeiras. NUPEM/ UFRJ, Macaé. Pp. 25-63.

Pereira, O.J. \& Assis, A.M. 2004. Fitossociologia da vegetação arbustiva fechada inundável de restinga no município de Linhares - ES. In: Anais do VI Simpósio de Ecossistemas Brasileiros: Patrimônio Ameaçado. Vol. 2. ACIESP, São José dos Campos. Pp. 407- 413.

Pereira, O.J. \& Gomes, J.M.L. 1993. Levantamento florístico das comunidades vegetais de restinga no município de Conceição da Barra/ES. In: Anais III Simpósio de Ecossistemas da Costa Brasileira. ACIESP, São Paulo. Pp. 67-78.

Pereira, O.J. 1990. Caracterização fitofisionômica da restinga de Setiba, Guarapari, ES. In: Anais Do II Simpósio de Ecossistemas da Costa Sul e Sudeste Brasileira: Estrutura, Função e Manejo. Vol. 3. ACIESP, Águas de Lindóia. Pp. 207-219.

Pereira, O.J.; Assis, A.M. \& Quinino, M.K. 2004. Estrutura da formação arbustiva aberta não inundável de restinga sobre terrenos Pleistocênicos - Linhares (ES). In: Anais Do VI Simpósio de Ecossistemas Brasileiros: Patrimônio Ameaçado. Vol. 2. ACIESP, São José dos Campos. Pp. 399-406.
Pereira, O.J.; Assis, A.M. \& Souza, R.L.D. 1998. Vegetação da restinga de Pontal do Ipiranga, Município de Linhares (ES). In: Anais do IV Simpósio de Ecossistemas Brasileiros. Vol. 3. ACIESP, São Paulo. Pp. 117-128.

Ribas, L.A.; Hay, J.D. \& Caldas-Soares, J.F. 1993. Moitas de restinga: ilhas ecológicas? In: Anais III Simpósio de Ecossistemas da Costa Brasileira. ACIESP, São Paulo. Pp. 79-88.

Sá, C.F.C. \& Araujo, D.S.D. 2009. Florística e estrutura de uma floresta restinga em Ipitangas, Saquarema, Rio de Janeiro, Brasil. Rodriguésia 60: 147-170.

Scarano, F.R.A. 1998. Comparison of dispersal, germination and establishment of woody plants subjected to distinct flooding regimes in Brazilian flood-prone forests and estuarine vegetation. In: Scarano, F.R. \& Franco, A.C. (eds.). Ecophysiological strategies of xerophytic and amphibious plants in the neotropics. Vol. 4. Series Oecologia Brasiliensis, Rio de Janeiro. Pp. 177-194.

Scherer, A.; Maraschin-Silva, F. \& Baptista, L.R.M. 2005. Florística e estrutura do componente arbóreo de matas de restinga arenosa no Parque Estadual de Itapuã, RS, Brasil. Acta Botanica Brasilica 19: 717-727.

Smith, A.R.; Pryer, K.M.; Schuettpel, E.; Korall, P.; Schneider, H. \& Wolf, P.G. 2006. A classification for extant ferns. Taxon 55: 705-731.

Suguio, K. \& Tessler, M.G. 1984. Planícies de cordões litorâneos Quaternários do Brasil: origem e nomenclatura. In: Lacerda, L.D.; Araujo, D.S.D.; Cerqueira, R. \& Turc, B. (orgs.). Restingas: origem, estrutura e processos. CEUFF, Niterói. Pp. 15-25.

Suguio, K.; Martin, L. \& Dominguez, J.M.L. 1982. Evolução do delta do rio Doce (ES) durante o quaternário: influência das variações do nível do mar. In: Suguio, K.; Meis, M.R. M. \& Tessler, M.G. (eds.). Atas IV Simpósio Quaternário do Brasil. Sociedade Brasileira de Geologia, Rio de Janeiro. Pp. 93-116.

Zaluar, H.T. \& Scarano, F.R. 2000. Facilitação em restingas de moitas: um século de buscas por espécies focais. In: Esteves, F.A. \& Lacerda, L.D. (orgs.). Ecologia de restingas e lagoas costeiras. NUPEM/UFRJ, Macaé. Pp. 3-23. 